\title{
A NUMERICAL LIAPUNOV-SCHMIDT METHOD WITH APPLICATIONS TO HOPF BIFURCATION ON A SQUARE
}

\author{
PETER ASHWIN, KLAUS BÖHMER, AND MEI ZHEN
}

Dedicated to Professor Dr. Karl Nickel on the occasion of his 70th birthday

\begin{abstract}
We discuss an iterative method for calculating the reduced bifurcation equation of the Liapunov-Schmidt method and its numerical approximation. Using appropriate genericity assumptions (with symmetry), we derive a Taylor series for the reduced equation, where the bifurcation behavior is determined by its numerical approximation at a finite order of truncation.

This method is used to calculate reduced equations at Hopf bifurcation of the two-dimensional Brusselator equations on a square with Neumann and Dirichlet boundary conditions. We examine several Hopf bifurcations within the threeparameter space. There are regions where we observe direct bifurcation to branches of periodic solutions with submaximal symmetry.
\end{abstract}

\section{INTRODUCTION}

Let $E \hookrightarrow \hat{E}$ be Banach spaces embedded into a Hilbert space with scalar product $\langle\cdot, \cdot\rangle$ and $G: E \times \mathbb{R} \rightarrow \hat{E}$ be a smooth mapping, equivariant under some action of a symmetry group $\Gamma$. We consider steady state and Hopf bifurcation of the problem

$$
\frac{\partial u}{\partial t}=G(u, \lambda) \text { with }(u, \lambda) \in E \times \mathbb{R}
$$

at a given bifurcation point $\left(u_{0}, \lambda_{0}\right)$. It is well known, in the case of steady state bifurcation with $\operatorname{Ker}\left(D G\left(u_{0}, \lambda_{0}\right)\right)$ finite-dimensional, that the LiapunovSchmidt method can be used to reduce equation (1) to a finite-dimensional bifurcation equation

$$
B(\alpha, \lambda)=0
$$

under certain assumptions on $D G\left(u_{0}, \lambda_{0}\right)$ and its kernel. Usually, these equations are highly nonlinear and an analysis of their solution can be rather difficult. However, in the neighborhood of the bifurcation point we can truncate the Taylor series of the equation at a sufficiently high order to give the bifurcation scenario. In $\S 2$ we consider a symmetry-respecting discretization of the

Received by the editor December 30, 1992 and, in revised form, June 28, 1993.

1991 Mathematics Subject Classification. Primary 65J15, 65N12, 58G28; Secondary 35B32.

Key words and phrases. Hopf bifurcation, steady state bifurcation, Liapunov-Schmidt method, finite determinacy, equivariant operator. 
Liapunov-Schmidt method to calculate the approximate bifurcation equation

$$
B^{h}(\alpha, \lambda)=0 \text {, }
$$

with $h$ the discretization parameter, up to any desired order of truncation of the Taylor series. In particular, we use assumptions of stability and finite determinacy of the exact bifurcation equations (incorporating any symmetries of the problem) to give equivalence of the true and approximate bifurcation scenarios (see also Ashwin, Böhmer, and Mei [9]).

For the rest of this section, we state the necessary definitions and introduce the concepts of germs and singularities of bifurcation equations, their stability and determinacy. We also introduce an iterative Liapunov-Schmidt reduction for Hopf bifurcation (thinking of it as a steady state bifurcation in loop space) and prove a theorem stated in [9] giving convergence of the iterative method. In $\S 2$ we discuss and also prove convergence of a suitable discretization of the iterative Liapunov-Schmidt method. This gives an extension of the possible equations to which we can practically apply the Liapunov-Schmidt reduction method. Finally, in $\S 3$, we examine applications of the method to computing Hopf bifurcations of the two-dimensional Brusselator equations on a square, with both Neumann and Dirichlet boundary conditions. We show that the bifurcation structure can be determined by a hybrid numerical/analytical/computeralgebraic method in cases where a purely analytical method fails to give results. We verify the calculations for Neumann boundary conditions by comparison with exact results obtained in Ashwin and Mei [10]. The details of the calculations are to be found in the Appendix (in the Supplement section at the end of this issue).

1.1. Preliminaries. The natural language for local steady state bifurcation problems is that of germs of functions and their singularities, see, e.g., Martinet [27], Golubitsky et al. [21, 23]. We consider 1-parameter problems in $m$ state variables with a bifurcation at $(0,0)$. If $E_{m, 1}^{\nu}$ is the module of germs at $(0,0)$ of $C^{\infty}$ vector- and matrix-valued functions from $\mathbb{R}^{m} \times \mathbb{R} \rightarrow \mathbb{R}^{\nu}(\nu=m, m \times m$, respectively) over the ring $E_{m, 1}^{1}$ of scalar functions, we define the set of bifurcation problems as

$$
\mathscr{F}:=\left\{f \in E_{m, 1}^{m}: f(0,0)=0, \partial_{u} f(0,0)=0, \partial_{\lambda} f(0,0)=0\right\} .
$$

The set of germs of $\Gamma$-equivariant bifurcation equations is defined as

$$
\mathscr{F}:=\{f \in \mathscr{F}: \gamma f(u, \lambda)=f(\gamma u, \lambda) \forall \gamma \in \Gamma\} .
$$

We have implicitly assumed that the symmetry group $\Gamma$ is represented orthogonally on $\mathbb{R}^{m}$. To decide when two bifurcation equations are merely deformations of each other, we need to define an equivalence relation on the space $\mathscr{F}$.

Definition 1. Two germs $f, g$ in $\mathscr{F}$ are equivalent (we write $f \sim g$ ) if and only if there exist germs of functions $X, \Lambda, S$ such that

$$
f(u, \lambda)=S(u, \lambda) g(X(u, \lambda), \Lambda(\lambda)),
$$


where

$$
\begin{aligned}
& X(0,0)=0, \Lambda(0)=0, \Lambda^{\prime}(0)>0, \\
& X(\gamma u, \lambda)=\gamma X(u, \lambda), S(\gamma u, \lambda) \gamma=\gamma S(u, \lambda) \forall \gamma \in \Gamma \text {, } \\
& \left.\begin{array}{l}
\partial_{u} X(0,0) \\
S(0,0)
\end{array}\right\} \text { are invertible linear maps with positive determinant. }
\end{aligned}
$$

Obviously, if $(X(u, \lambda), \Lambda(\lambda))$ and its inverse are smooth in some neighborhood of $(0,0)$, this is the definition of Golubitsky et al. [23]. The definition means that if $f$ and $g$ are equivalent, there is a locally invertible change of parameters $\Lambda(\lambda)$ such that $f(u, \lambda)$ and $g(X(u, \lambda), \Lambda(\lambda))$ have the same "number of zeros". The germ $g \in \mathscr{F}_{\Gamma}$ is said to be finitely determined if $j_{k} g \sim g$ for some $k \in \mathbb{N}$. (The symbol $j_{k} g$, called the $k$-jet, means the truncation of the Taylor expansion of $g$ with respect to all its arguments up to and including homogeneous terms of order $k$.) We say $g$ is $k$-determined if $g \sim j_{k} g$ and $g \not j_{l} g$ for all $l<k$.

For any $g \in \mathscr{F}_{\Gamma}$ we define the pseudonorm $\|\cdot\|_{k}^{0}$ (a norm on the subspace of $k$-jets) by

$$
\|g\|_{k}^{0}=\sum_{|i|+j \leq k}\left|\frac{\partial^{|i|+j} g}{\partial u^{i} \partial \lambda^{j}}(0,0)\right|,
$$

using a multi-index $i$.

Assumption. For the rest of this paper, we will assume that the germ of the reduced bifurcation equation $g \in \mathscr{F}_{\Gamma}$ is $k$-determined for some $k$, and is stable with respect to the pseudonorm (3). That is, there exists $\varepsilon>0$ such that all perturbations $f \in \mathscr{F}_{\Gamma}$ of $g$ with $\|f-g\|_{k}^{0}<\varepsilon$ satisfy $f \sim g$.

In [9], we demonstrate that for a $k$-determined bifurcation problem, stability with respect to $\|\cdot\|_{k}^{0}$ is equivalent to stability with respect to the Sobolev norm $\|\cdot\|_{k}$. One consequence of this assumption is that $\Gamma$ must be absolutely irreducibly represented on $\mathbb{R}^{m}$ (see [23]). This assumption is reasonable from the point of view of structural stability, and could also be expressed by saying that $g$ is its own universal unfolding. If a bifurcation comes from a model of a physical problem where we cannot expect to know the equations and parameters exactly, we can only get sensible results if our model is insensitive to small changes in these parameter values. In practice, we cannot tell if a problem is finitely determined and stable unless we can perform Liapunov-Schmidt reduction analytically, in which case we do not need a numerical method! However, we can verify if numerical approximations to coefficients in the Taylor expansion tend to values where the assumption would be broken, e.g., certain coefficients being zero might imply degenerate behavior.

We could also have chosen a weaker equivalence relation. For example, we could permit coordinate changes of the form $\Lambda(x, \lambda)$. This would be quite adequate if we only wish to distinguish branches and not their direction of branching in parameter space (this might be sufficient for branch switching of a path-following algorithm). Note that by choosing this more general equivalence, we get lower levels of determinacy; for example the pitchfork $x^{3}+x \lambda=0$ is three-determined for bifurcation equivalence but the truncation at second order $x \lambda=0$ is two-determined for the weaker equivalence. 
1.1.1. Symmetry. As demonstrated by Golubitsky et al. [21, 23], we need to include the symmetry of the problem in the reduction in order to obtain an equivariant reduced problem. For finite groups, a symmetry-respecting discretization can be defined as described below. For the case of continuous groups, this is much harder to achieve except in special cases. In general, we need to choose a basis for the discretization that contains its own group orbit, e.g., Fourier series for periodic problems.

If we miss some symmetry in the discretization of a symmetric problem, then naturally we cannot expect to get the actual bifurcation scenario reflected in the approximate one. Instead we should expect to see a deformation of it.

1.1.2. Consistent differentiability of the discretization. The above assumptions of stability and finite determinacy require that a few of the low-order terms of the Taylor expansion determine the bifurcation structure. To guarantee that the discretization reflects this behavior, we have to require that the derivatives of the discretized equation up to $k$ th order are $O\left(h^{p}\right)$ close to those of the true equations. This property is called consistent differentiability up to order $k$ and is discussed in [9], Böhmer et al. [11-13] and in $§ 2$.

As we are concerned with Hopf bifurcation, we first state a standard method (e.g., Vanderbauwhede [36]) for reducing the problem to a steady state problem.

1.1.3. Hopf bifurcation. We assume that the linearization $\partial_{u} G(0,0)$ of equation (1) has a pair of semisimple imaginary eigenvalues $\pm i \omega_{0}$, each of multiplicity $l$, and no other eigenvalues at $m i \omega_{0}$ for $m \in \mathbb{Z}, m \neq \pm 1$, where $l$ is a dimension of an absolutely irreducible representation of $\Gamma$. We define the following problem from the original 1-parameter problem:

$$
\Phi(u, \tau, \lambda):=G(u, \lambda)-\omega_{0}(1+\tau) \partial_{s} u
$$

as a mapping from $C_{2 \pi}^{1} \times \mathbb{R} \times \mathbb{R}$ to $\hat{C}_{2 \pi}$ with a trivial solution, where

$$
\begin{aligned}
& \hat{C}_{2 \pi}:=\{u(\cdot) \in C(\mathbb{R}, \hat{E}): u(s+2 \pi)=u(s)\} \text { and } \\
& C_{2 \pi}^{1}:=\left\{u(\cdot), \frac{\partial u}{\partial s}(\cdot) \in C(\mathbb{R}, E): u(s+2 \pi)=u(s)\right\} .
\end{aligned}
$$

We treat the parameter $\tau$ as a state variable (i.e., we allow changes of coordinates mixing $\tau$ and $u(t)$ ), but we keep $\lambda$ as the parameter. We use the usual inner product

$$
\langle u(s), v(s)\rangle_{C_{2 \pi}^{i}}:=\frac{1}{2 \pi} \int_{s=0}^{2 \pi} \sum_{j=0}^{i}\left\langle\frac{\partial^{j} u}{\partial s^{j}}, \frac{\partial^{j} v}{\partial s^{j}}\right\rangle d s .
$$

Using the hypotheses for Hopf bifurcation, we find that $D \Phi(0,0)$ satisfies the conditions for Liapunov-Schmidt reduction: fixed points in $C_{2 \pi}^{1}$ correspond to periodic solutions of equation (1).

1.2. The Liapunov-Schmidt reduction. Consider the operator equation

$$
\begin{aligned}
& \Phi: E \times \mathbb{R} \rightarrow \hat{E}, \\
& 0=\Phi(u, \lambda)=: D \Phi(0,0)(u, \lambda)+R(u, \lambda),
\end{aligned}
$$

where $\Phi$ is a smooth 1-parameter mapping between the Banach spaces $E \hookrightarrow \hat{E}$ with $\Phi(0,0)=0$ (in fact we only need one degree of smoothness greater than 
the determinacy). Note that by definition, $R(z)=O\left(\|z\|^{2}\right)$, where $z=(u, \lambda)$. We require that

$$
\Phi_{0}^{\prime}:=D \Phi(0,0):=\left(\partial_{u} \Phi(0,0), \partial_{\lambda} \Phi(0,0)\right)
$$

is a Fredholm operator of index 1 with a kernel of dimension $m+1, m \geq 1$. Furthermore, we exclude turning point bifurcations (they are regular points of the mapping $\Phi)$ and so can assume, reparameterizing if necessary, that

$$
\partial_{\lambda} \Phi(0,0)=0
$$

This corresponds to the assumption of a trivial solution from which the branches bifurcate.

Using the Fredholm condition, we split the spaces in the standard way:

$$
\begin{aligned}
& E \times \mathbb{R}=\operatorname{Ker}\left(\Phi_{0}^{\prime}\right) \oplus \operatorname{Im}\left(\Phi_{0}^{\prime *}\right), \\
& \hat{E}=\operatorname{Ker}\left(\Phi_{0}^{\prime *}\right) \oplus \operatorname{Im}\left(\Phi_{0}^{\prime}\right),
\end{aligned}
$$

with $\Phi_{0}^{\prime *}$ the adjoint operator and with $\oplus$ indicating orthogonality with respect to $\langle\cdot, \cdot\rangle$, usually the inner product in $L^{2}(\cdot)$ of the original Hilbert space into which $E$ and $\hat{E}$ are embedded. We define the projections

$$
\begin{aligned}
& Q: E \times \mathbb{R} \rightarrow \operatorname{Im}\left(\Phi_{0}^{\prime *}\right), \operatorname{Ker}(Q)=\operatorname{Ker}\left(\Phi_{0}^{\prime}\right)=\operatorname{Ker}\left(\partial_{u} \Phi_{0}\right) \times \mathbb{R} \text { and } \\
& \hat{Q}: \hat{E} \rightarrow \operatorname{Im}\left(\Phi_{0}^{\prime}\right), \operatorname{Ker}(\hat{Q})=\operatorname{Ker}\left(\Phi_{0}^{\prime *}\right) .
\end{aligned}
$$

Using these projectors, we write $z=\eta+w$ with $\eta \in \operatorname{Ker}\left(\Phi_{0}^{\prime}\right)$ and $w \in \operatorname{Im}\left(\Phi_{0}^{\prime *}\right)$ and split equation (5) into two:

$$
\begin{aligned}
& \hat{Q} \Phi(\eta+w)=0, \\
& (I-\hat{Q}) \Phi(\eta+w)=0
\end{aligned}
$$

Equation (7) is solvable in terms of $\eta$ for small $\eta$ and gives $w(\eta)=O\left(\eta^{2}\right)$ uniquely, and this is substituted into equation (8) to give the reduced bifurcation equation:

$$
B(\eta):=(I-\hat{Q}) \Phi(\eta+w(\eta))=(I-\hat{Q}) R(\eta+w(\eta))=0 .
$$

In order for symmetries of $\Phi$ to be inherited by $B$, we must choose projectors $Q, \hat{Q}$ commuting with the symmetries, see e.g., [21] or [36]. We now give an iterative method to solve equation (7) that develops the Taylor expansion of $B$ and is presented in [6-9]. This was used in [1] to calculate higher-order terms in a periodic boundary value problem. The functions $w(\eta)$ and $B(\eta)$ can be obtained iteratively using the following Algorithm.

\section{Algorithm 2. Truncated Liapunov-Schmidt Method.}

Initialization. Pick an initial function

$$
w_{1}(\eta)=0
$$

a mapping from $\operatorname{Ker}\left(\Phi_{0}^{\prime}\right)$ to $\operatorname{Im}\left(\Phi_{0}^{\prime *}\right)$. We identify $\eta \in \operatorname{Ker}\left(\Phi_{0}^{\prime}\right)$ with $(\alpha, \lambda) \epsilon$ $\mathbb{R}^{m+1}$ and $\operatorname{Ker}\left(\Phi_{0}^{\prime *}\right)$ with $\mathbb{R}^{m}$. 
Iteration. For $k=2,3, \ldots$ until determinacy, do: at each stage define the truncated bifurcation equations to be

$$
B_{k}(\eta):=(I-\hat{Q}) j_{k} R\left(\eta+w_{k-1}(\eta)\right)=0,
$$

where $B_{k}$ is a polynomial of $k$ th order from $\operatorname{Ker}\left(\Phi_{0}^{\prime}\right)$ to $\operatorname{Ker}\left(\Phi_{0}^{\prime *}\right)$, and

$$
w_{k}(\eta):=-Q\left(\Phi_{0}^{\prime}\right)^{-1} \hat{Q} j_{k} R\left(\eta+w_{k-1}(\eta)\right) .
$$

This iteration creates a sequence of $k$ th-order polynomial functions

$$
w_{k}: \operatorname{Ker}\left(\Phi_{0}^{\prime}\right) \rightarrow \operatorname{Im}\left(\Phi_{0}^{\prime *}\right) .
$$

Theorem 3. For the iteration defined in (11), (12), we have

$$
w_{k}(\eta)=j_{k} w(\eta) \text { and } B_{k}(\eta)=j_{k} B(\eta)
$$

Proof. Consider

$$
w_{k+1}-w_{k}=-Q\left(\Phi_{0}^{\prime}\right)^{-1} \hat{Q}\left[j_{k+1} R\left(\eta+w_{k}(\eta)\right)-j_{k} R\left(\eta+w_{k-1}(\eta)\right)\right]
$$

Now $j_{1}(R(\eta))=0$ implies that

$$
j_{k}(R(y(\eta)))=j_{k}\left(R\left(j_{k-1} y(\eta)\right)\right)
$$

for all functions $y(\eta)$ with $j_{0}(y(\eta))=1$. By induction we get that for all $k \geq 2$

$$
\begin{aligned}
& j_{k}\left(R \left(\eta+w_{k}(\eta)-R\left(\eta+w_{k-1}(\eta)\right)=0\right.\right. \\
\Rightarrow & j_{k}\left(w_{k+1}-w_{k}\right)=0 \\
\Rightarrow & j_{k} w_{k+1}=w_{k} .
\end{aligned}
$$

By the fact that $j_{k} Q \Phi\left(\eta+w_{k}(\eta)\right)=j_{k} Q \Phi(\eta+w(\eta))=0$, this means that the solution $w(\eta)$ of $Q R(\eta+w(\eta))=0$ has Taylor series $j_{k} w(\eta)=w_{k}(\eta)$. This implies that $j_{k} B(\eta)=B_{k}(\eta)$.

\section{DisCRETIZiNG THE TRUNCATED LiaPUNOV-SCHMIDT METHOD}

2.1. Finite and continuous symmetries. As described in Golubitsky and Stewart [22], at Hopf bifurcation we gain an extra $\mathbf{S}^{1}$ symmetry from the fact that our system is autonomous, and this may cause problems with the discretization. However, the simplicity of the dependence on the time derivative allows us to work in spaces of finite Fourier sums:

$$
P_{k}:=\left\{\sum_{m=0}^{k} a_{m} \cos m t+b_{m} \sin m t: a_{m}, b_{m} \in E\right\} .
$$

In fact, the calculation of $w_{k+1}$ and $B_{k+1}$ can be performed in $P_{2^{k}}$.

For other continuous symmetries, we can think of the whole group as split into the semidirect product of a continuous and a finite group $\Gamma=\Gamma_{c} \times_{s} \Gamma_{f}$. For many important problems, the continuous group can be represented as acting on a $\Gamma$-symmetry-respecting basis (e.g., Fourier series for $\mathbf{T}^{k}$, spherical harmonics for SO(3), see e.g. Dellnitz [16] and Gekeler [19]). In the case that finite products of elements in this basis can be expressed or approximated as finite sums of other elements in the basis, we can use finite bases to describe a discretization preserving the full symmetry. We therefore assume for the rest of 
this section that our problem is equivariant under some finite symmetry group $\Gamma$.

In order to preserve equivariance in the discretized iteration process, we use the technique developed in Böhmer [11,12]. We now give a short outline of necessary results adapted from [12].

2.2. Consistently differentiable discretization methods. We consider discretizations of (5) indexed by $h \in H$. We use linear bounded restriction operators $\pi^{h}$ and $\hat{\pi}^{h}$ mapping $E \times \mathbb{R}$ and $\hat{E}$ into the respective finite-dimensional discrete spaces $E^{h} \times \mathbb{R}$ and $\hat{E}^{h}$. We assume $\pi^{h}$ acts on $z=(u, \lambda) \in E \times \mathbb{R}$ by transforming $u \in E$ into a discrete $u^{h}=\pi^{h} u \in E^{h}$, leaving $\lambda$ unchanged. Let $\pi^{h}, \hat{\pi}^{h}$ satisfy:

$$
\begin{aligned}
& \pi^{h}: E \times \mathbb{R} \rightarrow E^{h} \times \mathbb{R}, \quad \hat{\pi}^{h}: \hat{E} \rightarrow \hat{E}^{h}, \\
& \pi^{h} z=\pi^{h}(u, \lambda)=\left(\pi^{h} u, \lambda\right) \text { and }\left\|\pi^{h} z\right\|=\|z\|+O\left(h^{p}\|z\|_{E_{s}}\right), \\
& \left\|\hat{\pi}^{h} \hat{y}\right\|=\|\hat{y}\|+O\left(h^{p}\|\hat{y}\|_{\hat{E}_{s}}\right)
\end{aligned}
$$

for fixed $z, \hat{y}$ in appropriate subspaces $E_{s}, \hat{E}_{s}$. The $O\left(h^{p}\right)$ term in $\pi^{h} z$ is due to $\pi^{h} u$. The operator $\Phi$ in (5) is transformed into a discrete operator $\Phi^{h}: \operatorname{domain}\left(\Phi^{h}\right) \subset E^{h} \rightarrow \hat{E}^{h}$. If $E$ is defined for a Sobolev norm with derivatives of order $|i|+j \leq n$, then $E_{s}$ requires a norm with derivatives of orders $|i|+j \leq n+p$. In such a case we choose $n$ such that $\Phi$ is continuous on $E$. For simplicity, we do not distinguish between $E, \hat{E}$ and their smooth subspaces $E_{s}, \hat{E}_{s}$. Strictly speaking, the latter are needed for consistency and convergence in (15) and (16), but these conditions may be relaxed by using weak discrete Sobolev norms as indicated in Hackbusch [26]. By expanding $\Phi$ in a Taylor series, we can ensure that, for many discretization methods, $\Phi^{h}$ is $r$ times consistently differentiable:

$$
\left\{\begin{array}{l}
\Phi^{h}\left(\pi^{h} z\right)=\hat{\pi}^{h} \Phi z+O\left(h^{p}\|z\|_{E_{s}}\right) \text { for } z \in E_{s} \text { and for } j=1, \ldots, r \\
\Phi^{h(j)}\left(\pi^{h} z\right) \pi^{h} z_{1} \cdots \pi^{h} z_{j}=\hat{\pi}^{h}\left[\Phi^{j}(z)+\varepsilon\left(h^{p}, z\right)\right] z_{1} \cdots z_{j} \\
\text { for } z, z_{1}, \ldots, z_{j} \in E_{s}
\end{array}\right.
$$

where $\varepsilon\left(h^{p}, z\right)$ represents a $j$-linear operator and

$$
\left\|\varepsilon\left(h^{p}, z\right)\right\| \leq O\left(h^{p}\|z\|_{E_{s}}\right) .
$$

Since the operators $\Phi^{h(j)}\left(\pi^{h} z\right) \pi^{h} \cdots \pi^{h}$ and $\hat{\pi}^{h} \Phi^{(j)}(z)$ in (16) act multilinearly on $z_{1}, \ldots, z_{j}$, the same is true for $\varepsilon(h, z)$ by definition. Example calculations showing this are described in [9]. A combination of (16) with the usual stability

$$
\left\|z^{h}-\bar{z}^{h}\right\| \leq C\left\|\Phi^{h}\left(z^{h}\right)-\Phi^{h}\left(\bar{z}^{h}\right)\right\|
$$

for $z^{h}, \bar{z}^{h}$ near the solution, yields the intended convergence.

2.3. Equivariant discretization methods. We choose discretization methods which preserve the $\Gamma$-equivariance of the original problem. In $\S 1$ we discussed the matter of reducing the problem to a finite-dimensional system with discrete symmetries and finite group $\Gamma$. Indicating the action of $\gamma \in \Gamma$ on elements in 
$E, \hat{E}, E^{h}$ and $\hat{E}^{h}$, respectively, with the same $\gamma$, we have

$$
\Phi(\gamma u, \lambda)=\gamma \Phi(u, \lambda) \text { for all } \gamma \in \Gamma .
$$

For the discretization $\Phi^{h}$ we have to choose $E^{h}$ and $\hat{E}^{h}$ such that, e.g., $u^{h} \in$ $E^{h}$ implies that $\gamma u^{h} \in E^{h}$ for all $\gamma \in \Gamma$. Furthermore, $\Phi^{h}$ and the restriction operators $\pi^{h}, \hat{\pi}^{h}$ must be defined such that they commute with the action of $\Gamma$ on the appropriate spaces, i.e.,

$$
\begin{aligned}
& \Phi^{h}\left(\gamma u^{h}, \lambda\right)=\gamma \Phi^{h}\left(u^{h}, \lambda\right), \\
& \pi^{h} \gamma u^{h}=\gamma \pi^{h} u^{h}, \hat{\pi}^{h} \gamma \hat{u}^{h}=\gamma \hat{\pi}^{h} \hat{u}^{h} \\
& \text { for all } \gamma \in \Gamma \text { and } u^{h} \in E^{h}, \hat{u}^{h} \in \hat{E}^{h} .
\end{aligned}
$$

This is achieved by approximating the $\Gamma$-equivariant terms of, e.g., function values, derivatives or integrals in an appropriate $\Gamma$-equivariant way. As examples, let us study the evaluation of $u$, the Laplacian $\Delta u$ and a function $g(\nabla u)$ for functions defined on a domain $\Omega \subset \mathbb{R}^{2}$ admitting a symmetry group $\Gamma$. The set of gridpoints $\mathbf{G}_{h}$, constituting $E^{h}$ (and $\hat{E}^{h}$ ), and the approximations $\Delta^{h}, \nabla^{h}$ and $u^{h}$ for $\Delta, \nabla$ and $u$ respectively, satisfy

$$
\begin{aligned}
& \gamma \mathbf{G}_{h}=\mathbf{G}_{h}, \Delta^{h} \gamma u^{h}=\gamma \Delta^{h} u^{h}, \\
& \nabla^{h} \gamma u^{h}=\gamma \nabla^{h} u^{h}, u^{h}(\gamma(x, y))=\gamma u^{h}(x, y) \forall \gamma \in \Gamma .
\end{aligned}
$$

Hence, we can use the five-point star approximation for finite difference approximation of the Laplacian $\Delta$ on $\Omega=[0,1]^{2}\left(\Gamma=\mathbf{D}_{4}\right)$, or the seven-point star for a regular hexagonal grid $\left(\Gamma=\mathbf{D}_{6}\right)$. For $g\left(\nabla^{h} u\right)$ or $u^{h}$ we must do approximations using these $\Gamma$-invariant stars. In this case we have convergence of order $p=2$. The discrete inner products, e.g. (22), have to be approximated with the same order, so for $\Omega=[0,1]^{2}$ a trapezoidal rule with appropriate weights on the boundary points will work.

In the case of finite elements, the finite-dimensional subspaces should be chosen equivariantly. If variational approaches to (17) are studied, the $\Gamma$ equivariance is directly inherited by the discretizations. If finitely many functionals are equal to zero, the corresponding discretizations must again be $\Gamma$ equivariant.

By these considerations we indicate how (17) can be equivariantly discretized. Detailed examples are studied in Allgower et al. [4, 5].

Since solutions of arbitrarily discretized $\Phi^{h}\left(u^{h}\right)=0$ will not respect any symmetries of the problem, we have to formulate $\Phi^{h}$ with respect to a basis maintaining the symmetry, a so-called symmetry-respecting basis; see StiefelFässler [31], Allgower, Böhmer, and Mei [4, 5], Allgower, Böhmer, Georg, and Miranda [3], Georg and Miranda [20] and Douglas and Mandel [17]. Then we are able to numerically define fixed point subspaces within $E^{h}$ and $\hat{E}^{h}$ :

$$
E^{h, \Sigma}:=\operatorname{Fix}^{\Sigma}\left(E^{h}\right):=\left\{z^{h} \in E^{h} \mid z^{h}=\sigma z^{h} \forall \sigma \in \Sigma\right\} .
$$

As a consequence of (18) we are able to consider the discrete problem $\Phi^{h}\left(z^{h}\right)$ on a fixed point subspace (19) of subgroups $\Sigma \preceq \Gamma$, i.e., we have that $\Phi^{h}$ and all its derivatives (and remainder terms) evaluated at $z^{h}$ are $\Sigma_{z^{h}}$ equivariant, with $\Sigma_{z^{h}}$ being the isotropy subgroup of the point $z^{h}$. If $\Phi^{h}$ is stable or $r$ times consistently differentiable, the same is true for $\Phi^{h, \Sigma}$. 
Throughout, we have assumed that we study the original problem in its singularity at $(0,0)$. The discretization $\Phi^{h}$ will usually not be singular at $(0,0)$, but at a slightly perturbed point, in particular, the bifurcation will occur at some $\lambda^{h} \approx 0$. Again, we use coordinate transformations to shift the singularity of $\Phi^{h}$ to $(0,0)$. Let $z, \varphi$ or $\hat{\psi}$ be solutions of

$$
\Phi(z)=0, \Phi^{\prime}(0,0) \varphi=g \text { or } \Phi^{\prime *}(0,0) \hat{\psi}=\hat{g} ;
$$

then there exist discrete solutions $z^{h}, \varphi^{h}, \hat{\psi}^{h}$ of

$$
\Phi^{h}\left(z^{h}\right)=0, \Phi^{h \prime}(0,0) \varphi^{h}=\pi^{h} g \text { or } \Phi^{h \prime *}(0,0) \hat{\psi}^{h}=\hat{\pi}^{h} \hat{g},
$$

see Allgower and Böhmer [2], such that

$$
\pi^{h} z-z^{h}=O\left(h^{p}\right), \pi^{h} \varphi-\varphi^{h}=O\left(h^{p}\right), \hat{\pi}^{h} \hat{\psi}-\hat{\psi}^{h}=O\left(h^{p}\right) .
$$

The $O\left(h^{p}\right)$ terms are also elements in $E^{h, \Sigma}$. For more details, see [9].

2.4. Equivariant discretization near singularities. We shall restrict our discussion to discretizations of bifurcation problems at $(0,0)$ with the following properties:

(a) $\partial_{\lambda} \Phi(0,0)=0$;

(b) The discretized problem has the identical symmetry and a bifurcation at $(0,0)$, usually after a shift;

(c) $\partial_{\lambda} \Phi^{h}(0,0)=0$;

(d) The kernels of $\Phi_{0}^{\prime}$ and $\Phi_{0}^{h^{\prime}}$ have the same dimension.

(Since the higher dimensions of these kernels are generically caused by symmetry, this and our assumption in $\S 1$ imply $20(\mathrm{~d})$.) We need the results in $\S \S 2.1$ and 2.2 to be able to formulate an appropriate discretization reflecting the bifurcation scenario close to singularities. This singularity may be analytically or numerically determined in the sense that we know either

$$
\operatorname{Ker}\left(\Phi_{0}^{\prime}\right)=\left[\psi_{1}, \ldots, \psi_{m}\right] \times \mathbb{R}, \operatorname{Ker}\left(\Phi_{0}^{\prime *}\right)=\left[\hat{\psi}_{1}, \ldots, \hat{\psi}_{m}\right]
$$

or approximations

$$
\operatorname{Ker}\left(\Phi_{0}^{h \prime}\right)=\left[\psi_{1}^{h}, \ldots, \psi_{m}^{h}\right] \times \mathbb{R}, \operatorname{Ker}\left(\Phi_{0}^{h \prime *}\right)=\left[\hat{\psi}_{1}^{h}, \ldots, \hat{\psi}_{m}^{h}\right]
$$

with

$$
\psi_{i}^{h}=\pi^{h} \psi_{i}+O\left(h^{p}\right), \hat{\psi}_{i}^{h}=\hat{\pi}^{h} \hat{\psi}_{i}+O\left(h^{p}\right) .
$$

Working with an equivariant discretization method, we may assume that $\psi_{i}^{h}$ and $\hat{\psi}_{i}^{h}$ have been determined in the same fixed point subspaces as the $\psi_{i}$ and $\hat{\psi}_{i}$ respectively. Furthermore, our assumption that the problem is a generic $\Gamma$-equivariant problem means that the kernel of the linearized operator is of a generic size, i.e., the dimension of an absolutely irreducible representation of $\Gamma$ for the case of one parameter.

In $\S 1$ we described a truncated Liapunov-Schmidt method, defining the $B_{k}$ and $w_{k}$. By means of the properties of the operator $\Phi$, the projections $Q$ and $\hat{Q}$ have to be transformed into a discrete setting. We now discuss these discrete projectors $Q^{h}$ and $\hat{Q}^{h}$. 
A discrete realization of Algorithm 2 is prevented by the fact that the usual stability of $\Phi^{h}$, and hence convergence, breaks down near singularities. This difficulty is studied in Böhmer [11, 12] and Böhmer and Mei [13]. However, the situation here is much simpler, owing to the assumption of genericity in the (symmetric) discrete setting. In [11] and [13], we had to use extension operators for the case of $\operatorname{Ker}\left(\Phi_{0}^{\prime}\right)$ being of a different dimension than $\operatorname{Ker}\left(\Phi_{0}^{h \prime}\right)$. We use the discrete pairing or inner product $\langle\cdot, \cdot\rangle^{h}$ related to $\langle\cdot, \cdot\rangle$ by

$$
\left\langle\pi^{h} u, \pi^{h} v\right\rangle^{h}=\langle u, v\rangle+O\left(h^{p}\|u\|_{s}\|v\|_{s}\right),
$$

where $\|\cdot\|_{s}$ indicates the smooth norm. Corresponding results hold in $\hat{E}$ and $\hat{E}^{h}$ as well. We assume that the original and discrete pairings $\langle\cdot, \cdot\rangle$ and $\langle\cdot, \cdot\rangle^{h}$ are $\Gamma$-invariant,

$$
\langle u, v\rangle=\langle\gamma u, \gamma v\rangle,\left\langle u^{h}, v^{h}\right\rangle^{h}=\left\langle\gamma u^{h}, \gamma v^{h}\right\rangle^{h} \forall \gamma \in \Gamma,
$$

by defining, if necessary, a symmetrized version

$$
[u, v]:=\frac{1}{|\Gamma|} \sum_{\gamma \in \Gamma}\langle\gamma u, \gamma v\rangle
$$

for the finite group $\Gamma$, where $|\Gamma|$ is the order of $\Gamma$. We define

$$
\begin{aligned}
& E^{h}=\operatorname{Ker}\left(\Phi_{0}^{h \prime}\right) \oplus \operatorname{Im}\left(\Phi_{0}^{h / *}\right)=: N^{h} \oplus M^{h}, \\
& \hat{E}^{h}=\operatorname{Ker}\left(\Phi_{0}^{h \prime *}\right) \oplus \operatorname{Im}\left(\Phi_{0}^{h \prime}\right)=: \hat{N}^{h} \oplus \hat{M}^{h} .
\end{aligned}
$$

Since all the kernels and images in (24) are $\Gamma$-invariant subspaces, the orthogonality is also valid for the $\Gamma$-invariant pairing $\langle\cdot, \cdot\rangle^{h}$. The next proposition relates the continuous and discrete projectors, and is stated without proof in [9]. Consider

$$
\begin{array}{ll}
Q u:= & u-\sum_{i=1}^{m}\left\langle\psi_{i}, u\right\rangle \psi_{i}, \\
& Q(u, \lambda)=Q u \text { for }(u, \lambda) \in E \times \mathbb{R}, \\
\hat{Q} \hat{u}:= & \hat{u}-\sum_{i=1}^{m}\left\langle\hat{\psi}_{i}, \hat{u}\right\rangle \hat{\psi}_{i} \text { for } \hat{u} \in \hat{E}, \\
Q^{h} u^{h}:= & u^{h}-\sum_{i=1}^{m}\left\langle\psi_{i}^{h}, u^{h}\right\rangle^{h} \psi_{i}^{h}, \\
& Q^{h}\left(u^{h}, \lambda\right):=Q^{h} u^{h} \text { for }\left(u^{h}, \lambda\right) \in E^{h} \times \mathbb{R}, \\
\hat{Q}^{h} \hat{u}^{h}:= & \hat{u}^{h}-\sum_{i=1}^{m}\left\langle\hat{\psi}_{i}^{h}, \hat{u}^{h}\right\rangle^{h} \hat{\psi}_{i}^{h} \text { for all } \hat{u}^{h} \in \hat{E}^{h} .
\end{array}
$$

Proposition 4. Under the assumption that (21)-(23) are satisfied, the projectors $Q, \hat{Q}, Q^{h}, \hat{Q}^{h}$ are $\Gamma$-equivariant projections satisfying

$$
\begin{aligned}
& \pi^{h} Q z-Q^{h} \pi^{h} z=O\left(h^{p}\|z\|\right), \\
& \hat{\pi}^{h} \hat{Q} \hat{z}-\hat{Q}^{h} \hat{\pi}^{h} \hat{z}=O\left(h^{p}\|\hat{z}\|\right),
\end{aligned}
$$

with the error terms also equivariant under $\Gamma$. The same is true for the projection $I-Q$.

Proof. Since, for each $\gamma \in \Gamma$, the $\left\{\gamma \psi_{i}\right\}_{i=1}^{m}$ represent a basis for $\operatorname{Ker}\left(\partial_{u} \Phi_{0}\right)$, we have

$$
Q \gamma u=\gamma u-\sum_{i=1}^{m}\left\langle\psi_{i}, \gamma u\right\rangle \psi_{i}=\gamma u-\sum_{i=1}^{m}\left\langle\gamma \psi_{i}, \gamma u\right\rangle \gamma \psi_{i}=\gamma Q u
$$


and so on. Furthermore,

$$
\begin{array}{r}
\pi^{h} Q u-Q^{h} \pi^{h} u=\sum_{i=1}^{m}\left(\left(\left\langle\psi_{i}, u\right\rangle-\left\langle\psi_{i}^{h}, \pi^{h} u\right\rangle^{h}\right) \pi^{h} \psi_{i}\right. \\
\left.+\left\langle\psi_{i}^{h}, \pi^{h} u\right\rangle^{h}\left(\pi^{h} \psi_{i}-\psi_{i}^{h}\right)\right)=O\left(h^{p}\right) .
\end{array}
$$

Summarizing, we have discrete operators that are close to $\Phi, Q$ and $\hat{Q}$ up to $\Gamma$-equivariant perturbations of (relative) size $O\left(h^{p}\right)$.

To obtain stability of the numerical solutions near the singularity in (11), (12), it is necessary to modify the conventional stability by assuming that

$$
\left.\Phi_{0}^{h \prime}\right|_{M^{h}}: M^{h} \rightarrow \hat{M}^{h}:=\Phi_{0}^{h \prime}\left(M^{h}\right)
$$

is boundedly invertible, uniformly for small $h$, in the appropriate norms. Results of this type have been obtained for discretized problems by Brezzi, Rappaz, and Raviart [14], Esser [18], Grigorieff [25], Reinhard [30], Stummel [32, 33], and Vainikko [35]. To get the $k$-jets, $j_{k}$, for the discrete problem, we merely truncate the Taylor series for the discrete operators. The numerical version of the iterative Liapunov-Schmidt algorithm follows.

\section{Algorithm 5. Discrete Truncated Liapunov-Schmidt Method.}

Initialization. Define the function $w_{1}^{h}: \operatorname{Ker}\left(\Phi_{0}^{h \prime}\right) \rightarrow \operatorname{Im}\left(\Phi_{0}^{h / *}\right)$ by

$$
w_{1}\left(\eta^{h}\right)=0
$$

for all $\eta^{h} \in \operatorname{Ker}\left(\Phi_{0}^{h \prime}\right)$.

Iteration. For $k=2,3, \ldots$ until determinacy, do:

$$
B_{k}^{h}\left(\eta^{h}\right):=\left(I-\hat{Q}^{h}\right) j_{k} R^{h}\left(\eta^{h}+w_{k-1}^{h}\left(\eta^{h}\right)\right),
$$

where $B_{k}^{h}$ is a polynomial of $k$ th order in $\eta^{h}$ from $\operatorname{Ker}\left(\Phi_{0}^{h \prime}\right)$ to $\operatorname{Ker}\left(\Phi_{0}^{h / *}\right)$. We generate the next function $w_{k}^{h}$ with the formula

$$
w_{k}^{h}\left(\eta^{h}\right):=-Q^{h}\left(\Phi_{0}^{h \prime}\right)^{-1} \hat{Q}^{h} j_{k} R^{h}\left(\eta^{h}+w_{k-1}^{h}\left(\eta^{h}\right)\right)
$$

where $w_{k}^{h}$ is a $k$ th-order polynomial from $\operatorname{Ker}\left(\Phi_{0}^{h \prime}\right)$ to $\operatorname{Im}\left(\Phi_{0}^{h \prime *}\right)$.

The following result shows the required convergence of the discrete approximations to the true bifurcation equation. Thus, we are assured by the stability and $k$-determinacy of $B$ that (for small enough $h$ ) $B=0$ and $B_{k+1}^{h}=0$ give the same bifurcation scenario.

Theorem 6. Let $\Phi$ and its discretization $\Phi^{h}$ satisfy the following conditions:

(i) $r$ times consistent differentiability, see (16),

(ii) $\Phi^{h}$ is a $\Gamma$-equivariant discretization for $\Phi$, see (18);

(iii) the singularity satisfies (20) and (21);

(iv) the pairings are chosen to satisfy (22) and (23);

(v) $\Phi_{0}^{h \prime}$ satisfies the modified stability, see (25). 
Then the $w_{k}, B_{k}$ from Algorithm 2 and their discrete approximations from Algorithm 5 satisfy

(28) $\left\|w_{k}^{h}\left(\pi^{h} \eta\right)-\pi^{h} w_{k}(\eta)\right\|=O\left(h^{p}\|\eta\|\right),\left\|B_{k}^{h}\left(\pi^{h} \eta\right)-B_{k}(\eta)\right\|=O\left(h^{p}\|\eta\|\right)$

with the differences being $\Gamma$-equivariant. The norm for the $w_{k}^{h}$ approximation is given by (23); for $B_{k}^{h}$ we use (3). Furthermore, $B_{k}$ and $B_{k}^{h}$ are elements of $\mathscr{F}$.

Proof. We proceed by induction. Obviously, we have that, with the multi-index $\nu$,

$$
j_{k+1} R^{h}\left(\eta^{h}+w_{k}^{h}\left(\eta^{h}\right)\right)=j_{k+1}\left(\sum_{|\nu|=2}^{k+1} \frac{1}{\nu !} \Phi_{0}^{h(\nu)}\left(\eta^{h}+w_{k}^{h}\left(\eta^{h}\right)\right)^{\nu}\right) .
$$

In (29) and below in (30), (31), we may also omit all the discretization parameters $h$ to obtain the corresponding formulas for the original problem. To obtain (28), we have to compute the derivatives in (3) for $B_{k+1}(\eta)$ and $B_{k+1}^{h}\left(\pi^{h} \eta\right)$ as polynomials in $\eta$ of order $k+1$ with respect to $\eta$. Therefore, only derivatives up to order $k+1$ are of interest. Since the defining terms for $B_{k+1}$ and $B_{k+1}^{h}$, except $\eta, w_{k}(\eta)$ and $\eta^{h}, w_{k}^{h}\left(\eta^{h}\right)$, are independent of $\eta$ and $\eta^{h}$, respectively, see (6), (9), (11), (26), (27), (29), we exchange the derivatives $\frac{\partial^{i}}{\partial \eta^{i}}$, $\frac{\partial^{i}}{\partial\left(\eta^{h}\right)^{i}}$ of the relevant operators and need only study, for the multi-indices $i$ and $j$,

$$
\frac{\partial^{i}}{\left(\partial \eta^{h}\right)^{i}}\left(\eta^{h}+w_{k}^{h}\left(\eta^{h}\right)\right)^{j}, \quad 0 \leq|i|,|j| \leq k+1,
$$

where we identify $\eta \in \operatorname{Ker}\left(\Phi_{0}{ }^{\prime}\right)$ and $\eta^{h} \in \operatorname{Ker}\left(\Phi_{0}^{h^{\prime}}\right)$ with elements in $\mathbb{R}^{m+1}$.

For any $i=\left(i_{1}, \ldots, i_{m+1}\right), j=\left(j_{1}, \ldots, j_{m+1}\right)$ and $|i|,|j| \leq k+1$, we obtain

$$
\frac{\partial^{i}}{\partial \eta^{i}}(\eta)^{j}=\left\{\begin{array}{cl}
0 & \text { if } i_{l}>j_{l} \text { for at least one } l \\
j ! & \text { if } i=j, \\
\frac{j !}{(j-i) !} \eta^{j-i} & \text { if } i_{l} \leq j_{l} \text { for all } l .
\end{array}\right.
$$

In particular, we find with $\pi^{h} w_{1}=w_{1}^{h}=0$ and $\eta^{h}=\eta$, using (16) and Proposition 4, that

$$
\begin{aligned}
\frac{\partial^{i}}{\partial \eta^{i}}( & \left.B_{2}^{h}\left(\pi^{h} \eta\right)-B_{2}(\eta)\right) \\
= & \left(I-\hat{Q}^{h}\right) \frac{1}{2} \Phi_{0}^{h(2)} \pi^{h} \frac{\partial^{i}}{\partial \eta^{i}}(\eta)^{2}-(I-\hat{Q}) \frac{1}{2} \Phi_{0}^{(2)} \frac{\partial^{i}}{\partial \eta^{i}}(\eta)^{2} \\
= & \left(I-\hat{Q}^{h}\right) \frac{1}{2}\left(\Phi_{0}^{h(2)} \pi^{h}-\hat{\pi}^{h} \Phi_{0}^{(2)}\right) \frac{\partial^{i}}{\partial \eta^{i}}(\eta)^{2} \\
& +\left[\left(I-\hat{Q}^{h}\right) \hat{\pi}^{h}-(I-\hat{Q})\right] \frac{1}{2} \Phi_{0}^{(2)} \frac{\partial^{i}}{\partial \eta^{i}}(\eta)^{2} \\
= & O\left(h^{p}\|\eta\|\right) .
\end{aligned}
$$


Similarly, by (12), (27), (16), and Proposition 4,

$$
\begin{aligned}
\frac{\partial^{i}}{\left(\partial \eta^{h}\right)^{i}} & \left(w_{2}^{h}\left(\pi^{h} \eta\right)-w_{2}(\eta)\right) \\
= & Q^{h}\left(\Phi_{0}^{h^{\prime}}\right)^{-1} \hat{Q}^{h} j_{2} \frac{1}{2}\left(\Phi_{0}^{h(2)} \pi^{h}-\hat{\pi}^{h} \Phi_{0}^{(2)}\right) \frac{\partial^{i}}{\partial \eta^{i}}(\eta)^{2} \\
& +\left[Q^{h}\left(\Phi_{0}^{h^{\prime}}\right)^{-1} \hat{Q}^{h} j_{2} \hat{\pi}^{h}-Q\left(\Phi_{0}^{\prime}\right)^{-1} \hat{Q} j_{2}\right] \frac{1}{2} \Phi_{0}^{(2)} \frac{\partial^{i}}{\partial \eta^{i}}(\eta)^{2} \\
= & O\left(h^{p}\|\eta\|\right),
\end{aligned}
$$

where in both cases the $O\left(h^{p}\|\eta\|\right)$ terms are $\Gamma$-equivariant.

An inductive combination of (26), (27), (29)-(33) finally gives that $B_{k+1}^{h}$ is a small $\Gamma$-equivariant perturbation of $B_{k+1}$ in the pseudonorm (3). The corresponding estimates for derivatives of $w_{k+1}^{h}\left(\pi^{h} \eta\right)-w_{k+1}(\eta)$ with respect to $\eta$ are valid as well, but are not really important.

$B_{k+1}$ and $B_{k+1}^{h}$ are both $\Gamma$-equivariant since they are defined by $\Gamma$-equivariant operations based on the appropriate identification of $\eta=(\alpha, \lambda) \in$ $\mathbb{R}^{m+1}$. Hence, we only have to show that for $B_{k+1}$ and $B_{k+1}^{h}$ we have, for example,

$$
B_{k+1}(0,0)=0, \partial_{\alpha} B_{k+1}(0,0)=0, \partial_{\lambda} B_{k+1}(0,0)=0 .
$$

For $B_{k+1}$ and $B_{k+1}^{h}$, this is an immediate consequence of the fact that their linear terms are annihilated by the projection $(I-\hat{Q}) j_{k+1}$.

\section{HOPF BIFURCATION FOR THE 2-D BRUSSELATOR EQUATIONS}

In this section we consider an example application of the numerical LiapunovSchmidt technique to a Hopf bifurcation problem of a partial differential equation on a square domain. We consider an example from the dynamics of chemical systems. After discussing the linear problem and the symmetries of the nonlinear problem, we use null space coordinates in $\mathbb{C}^{2}$ giving two coupled cubic equations which generically determine the branching behavior. This normal form is investigated using results of Swift [34], and we obtain the bifurcation behavior at a variety of different spatial mode numbers, with both Neumann and Dirichlet boundary conditions.

3.1. The Brusselator equations. The following reaction-diffusion system proposed by Prigogine and Glansdorff [29] is a model for the Belousov-Zhabotinskii reaction; although it is not a very good model, it is simple enough to be amenable to analysis while showing a wide range of dynamical and bifurcation behavior:

$$
\begin{aligned}
& \partial_{t} u_{1}=\Delta u_{1}+A-(B+\lambda+1) u_{1}+u_{1}^{2} u_{2} \text { in } \Omega:=[0, \pi] \times[0, \pi] \\
& \partial_{t} u_{2}=d \Delta u_{2}+(B+\lambda) u_{1}-u_{1}^{2} u_{2},
\end{aligned}
$$

where $A, d \in(0,+\infty), B \in[0, \infty)$ are parameters. For convenience, we consider an extra parameter $\lambda \in \mathbb{R}$ as a bifurcation parameter. We take the boundary conditions to be Neumann $\left(\partial_{n} u_{1}=\partial_{n} u_{2}=0\right)$ or Dirichlet $\left(u_{1}=\right.$ $\left.A, u_{2}=(B+\lambda) / A\right)$ on $\partial \Omega$, where $\partial_{n}$ represents the normal derivative along 
the boundary of $\Omega$. It is easy to verify that this problem has a trivial solution

$$
\left(u_{1}, u_{2}\right)=(A,(B+\lambda) / A) .
$$

For simplicity, we fix the parameters $A, B$ and $d$ and consider bifurcation of equation (34) along the trivial solution curve with respect to $\lambda$. To this end, we substitute

$$
\left(u_{1}, u_{2}\right) \leftarrow(A,(B+\lambda) / A)+\left(u_{1}, u_{2}\right), t \leftarrow\left(\omega_{0}+\tau\right) t
$$

(the frequency $\omega_{0}$ is to be specified later) and derive the following equation from equation (34):

$$
\Phi(u, \lambda, \tau):=\left[L(B) u-\omega_{0} \partial_{t} u\right]+R(u, \lambda, \tau),
$$

where $u:=\left(u_{1}, u_{2}\right)^{T}$,

$$
L(B):=\left(\begin{array}{cc}
\Delta+B-1 & A^{2} \\
-B & d \Delta-A^{2}
\end{array}\right),
$$

and

$$
R(u, \lambda, \tau):=\left(\begin{array}{c}
1 \\
-1
\end{array}\right)\left(\frac{B+\lambda}{A} u_{1}^{2}+2 A u_{1} u_{2}+u_{1}^{2} u_{2}+\lambda u_{1}\right)-\tau \partial_{t} u .
$$

The operator $L$ maps from $E:=\left(C_{0}^{2, s}(\Omega)\right)^{2}$ to $\hat{E}:=(C(\Omega))^{2}$, where $C_{0}^{2, s}(\Omega)$ consists of twice differentiable functions on $\Omega$ satisfying a Hölder condition and homogeneous Dirichlet or Neumann boundary conditions. In particular, we consider bases for $C_{2 \pi}^{1}, \hat{C}_{2 \pi}$, see $\S 1.1 .3$, with coefficients in $E, \hat{E}$, respectively, to be

$$
\{1, \cos l t, \sin l t: l=1,2, \ldots\} .
$$

Obviously, the operator $\Phi$ maps $C_{2 \pi}^{1}$ into $\hat{C}_{2 \pi}$ and

$$
\Phi(0, \lambda, \tau)=0 \text { for all } \lambda, \tau \in[0, \infty) .
$$

Considering eigenfunctions of the Laplacian $-\Delta$ on the unit square with Neumann or Dirichlet boundary conditions, one sees easily that if for a given constant $d_{0}>0$ the system

$$
\begin{aligned}
B-1-A^{2}-\left(1+d_{0}\right) c & =0, \\
(1+c)\left(A^{2}+d_{0} c\right)-d_{0} B c & >0
\end{aligned}
$$

has solutions $\left(A_{0}, B_{0}\right) \in \mathbb{R}_{+}^{2}$ for exactly one eigenvalue $c:=\left(k^{2}+l^{2}\right) \pi^{2}, k, l \in$ $\mathbb{N}$ of $-\Delta$, then the operator

$$
\partial_{u} \Phi_{0}:=\partial_{u} \Phi(0,0,0)=L\left(B_{0}\right)-\omega_{0} \partial_{t}
$$

with $\omega_{0}:=\sqrt{(1+c)\left(A_{0}^{2}+d_{0} c\right)-d_{0} B_{0} c}$ is singular. We assume that the eigenvalue $c$ has multiplicity 2 , and so the null space $\operatorname{Ker}\left(\partial_{u} \Phi_{0}\right)$ is 4-dimensional. 
Moreover, we can write

$$
\operatorname{Ker}\left(\partial_{u} \Phi_{0}\right)=\left[\psi_{1}, \psi_{2}, \psi_{3}, \psi_{4}\right]
$$

with

$$
\begin{aligned}
P & =\left(P_{i j}\right)_{i, j=1,2}:=\frac{1}{\omega_{0}}\left(\begin{array}{cc}
B_{0}-1-c & A_{0}^{2} \\
-B_{0} & -d_{0} c-A_{0}^{2}
\end{array}\right), \\
\psi_{1} & :=\left(e_{1} \cos t+P e_{1} \sin t\right) \phi_{k l}, \quad \psi_{2}:=\left(-P e_{1} \cos t+e_{1} \sin t\right) \phi_{k l}, \\
\psi_{3} & :=\left(e_{1} \cos t+P e_{1} \sin t\right) \phi_{l k}, \quad \psi_{4}:=\left(-P e_{1} \cos t+e_{1} \sin t\right) \phi_{l k},
\end{aligned}
$$

where

$$
e_{1}:=\left(\begin{array}{l}
1 \\
0
\end{array}\right), \quad e_{2}:=\left(\begin{array}{l}
0 \\
1
\end{array}\right)
$$

and for Dirichlet boundary conditions

$$
\phi_{k l}(x, y):=\sin k x \sin l y, \phi_{l k}(x, y):=\sin l x \sin k y,
$$

or for Neumann boundary conditions

$$
\phi_{k l}(x, y):=\cos k x \cos l y, \phi_{l k}(x, y):=\cos l x \cos k y,
$$

see [10]. The null space of the adjoint operator $\partial_{u} \Phi^{*}(0,0,0)$ is given by

$$
\operatorname{Ker}\left(\partial_{u} \Phi_{0}^{*}\right)=\left[\psi_{1}^{*}, \psi_{2}^{*}, \psi_{3}^{*}, \psi_{4}^{*}\right]
$$

with $P_{21}=-B_{0} / \omega_{0}$ and

$$
\begin{array}{ll}
\psi_{1}^{*}:=\frac{1}{P_{21}}\left(P^{T} e_{2} \cos t+e_{2} \sin t\right) \phi_{k l}, & \psi_{2}^{*}:=\frac{1}{P_{21}}\left(-e_{2} \cos t+P^{T} e_{2} \sin t\right) \phi_{k l}, \\
\psi_{3}^{*}:=\frac{1}{P_{21}}\left(P^{T} e_{2} \cos t+e_{2} \sin t\right) \phi_{l k}, & \psi_{4}^{*}:=\frac{1}{P_{21}}\left(-e_{2} \cos t+P^{T} e_{2} \sin t\right) \phi_{l k} .
\end{array}
$$

This gives

$$
\left\langle\psi_{i}^{*}, \psi_{j}\right\rangle=\delta_{i j}, \quad i, j=1, \ldots, 4
$$

with the $L^{2}$-product

$$
\langle u, v\rangle=\frac{1}{2 \pi} \int_{0}^{2 \pi}\left(\frac{4}{\pi^{2}} \int_{\Omega}(u, v) d x d y\right) d t
$$

in $\hat{C}_{2 \pi}$, where $(\cdot, \cdot)$ represents the Euclidean product in $\mathbb{R}^{2}$. Since $\partial_{u} \Phi_{0}$ is a Fredholm operator with index 0 and its zero eigenvalues are of semisimple type, we have the decompositions

(46) $\hat{C}_{2 \pi}=\operatorname{Ker}\left(\partial_{u} \Phi_{0}\right) \oplus \operatorname{Im}\left(\partial_{u} \Phi_{0}\right), C_{2 \pi}^{1}=\operatorname{Ker}\left(\partial_{u} \Phi_{0}\right) \oplus\left(\operatorname{Im}\left(\partial_{u} \Phi_{0}\right) \cap C_{2 \pi}^{1}\right)$

Define the $\mathbf{D}_{4} \times \mathbf{S}^{1}$-equivariant projection $\hat{Q}: C_{2 \pi} \rightarrow \operatorname{Im}\left(\partial_{u} \Phi_{0}\right)$ by

$$
\hat{Q} w:=w-\sum_{i=1}^{4}\left\langle\psi_{i}^{*}, w\right\rangle \psi_{i}, \quad w \in C_{2 \pi} .
$$

Equation (46) shows that $\hat{Q}$ is a projection from $C_{2 \pi}^{1}$ into $\operatorname{Im}\left(\partial_{u} \Phi_{0}\right) \cap C_{2 \pi}^{1}$. Moreover, $Q=\left.\hat{Q}\right|_{C_{2 \pi}^{1}}$. 
3.2. Equivariance of the equations. The symmetries of the domain together with time-translation give the problem a $\mathbf{D}_{4} \times \mathbf{S}^{1}$ equivariance. This will be utilized to reduce the computational work. The $\mathbf{D}_{4}$ action on $\Omega=[0, \pi] \times[0, \pi]$ is generated by action of the reflections $R$ and $S$,

$$
R(x, y)=(y, x), S(x, y)=(\pi-x, y) \forall(x, y) \in \Omega .
$$

The action of $\mathbf{D}_{4} \times \mathbf{S}^{1}$ on $C_{2 \pi}$ is given by

$$
(\delta, \theta)\left(\begin{array}{l}
u(x, y, t) \\
v(x, y, t)
\end{array}\right)=\left(\begin{array}{l}
u\left(\delta^{-1}(x, y), t-\theta\right) \\
v\left(\delta^{-1}(x, y), t-\theta\right)
\end{array}\right) \forall(\delta, \theta) \in \mathbf{D}_{4} \times \mathbf{S}^{1} .
$$

This action induces an action on $\mathbb{R}^{4}$, coordinates in the null space $\sum \alpha_{i} \psi_{i}$. Since

$$
\begin{aligned}
& S \phi_{k l}=(-1)^{k} \phi_{k l}, \quad R \phi_{k l}=\phi_{l k}, \\
& S \phi_{l k}=(-1)^{l} \phi_{l k}, R \phi_{l k}=\phi_{k l}
\end{aligned}
$$

hold for Neumann boundary conditions (and similarly for Dirichlet), the induced action of $\mathbf{D}_{4}$ on $\left(\alpha_{1}, \ldots, \alpha_{4}\right)$ is generated by

$$
S\left(\alpha_{1}, \alpha_{2}, \alpha_{3}, \alpha_{4}\right)^{T}=\left((-1)^{k} \alpha_{1},(-1)^{k} \alpha_{2},(-1)^{l} \alpha_{3},(-1)^{l} \alpha_{4}\right)^{T}
$$

and

$$
R\left(\alpha_{1}, \alpha_{2}, \alpha_{3}, \alpha_{4}\right)^{T}=\left(\alpha_{3}, \alpha_{4}, \alpha_{1}, \alpha_{2}\right)^{T} .
$$

The action of $\mathbf{S}^{1}$ induced on $\left(\alpha_{1}, \ldots, \alpha_{4}\right)$ is

$$
T_{\theta}\left(\begin{array}{l}
\alpha_{1} \\
\alpha_{2} \\
\alpha_{3} \\
\alpha_{4}
\end{array}\right):=\left(\begin{array}{cccc}
\cos \theta & \sin \theta & 0 & 0 \\
-\sin \theta & \cos \theta & 0 & 0 \\
0 & 0 & \cos \theta & \sin \theta \\
0 & 0 & -\sin \theta & \cos \theta
\end{array}\right)\left(\begin{array}{l}
\alpha_{1} \\
\alpha_{2} \\
\alpha_{3} \\
\alpha_{4}
\end{array}\right)
$$

In particular,

$$
T_{\pi} \alpha=-\alpha, T_{\pi / 2} \alpha=\left(\alpha_{2},-\alpha_{1}, \alpha_{4},-\alpha_{3}\right)^{T} .
$$

Hence, the symmetry group of the (reduced) bifurcation equation depends on the parity of $(k, l)$. We have the following possibilities (the genericity of which is discussed at length in Crawford [15]):

- Both $k, l$ are even, implying

$$
S \alpha=\alpha .
$$

Together with (49) we see that $\mathbf{D}_{4} \times \mathbf{S}^{1}$ acts reducibly on $\mathbb{R}^{4}$.

- Both $k, l$ are odd, implying

$$
S \alpha=-\alpha .
$$

Here we also have $\mathbf{D}_{4} \times \mathbf{S}^{1}$ acting reducibly on $\mathbb{R}^{4}$.

- $(k, l)=($ even, odd $)($ or $(k, l)=($ odd,even $))$, implying

$$
\left.S \alpha=\left(\alpha_{1}, \alpha_{2},-\alpha_{3},-\alpha_{4}\right) \text { (or } S \alpha=-\left(\alpha_{1}, \alpha_{2},-\alpha_{3},-\alpha_{4}\right)\right) \text {. }
$$

In this case $\mathbf{D}_{4} \times \mathbf{S}^{1}$ acts irreducibly on $\mathbb{R}^{4}$. 
Note that the parity of the modes can also have an important effect on the nonlinear terms in the normal form for steady state bifurcation, in particular in mode interactions (cf. Gomes [24], Crawford [15]). We limit our discussion to the third case, where we can use generic $\mathbf{D}_{4} \times \mathbf{S}^{1}$ bifurcation theory. Work is underway to apply this method to the other cases [8].

3.3. Results. The details of the calculations to produce $B_{3}$, the cubic truncation of the reduced equations, are given in the Appendix. Recall from Swift [34] that the normal form for $\mathbf{D}_{4}$ Hopf bifurcation is determined to cubic order by three complex coefficients $U, V, W$, and can be written

$$
0=z_{+}\left[\mu+i \phi+U\left(\left|z_{+}\right|^{2}+\left|z_{-}\right|^{2}\right)+V\left|z_{+}\right|^{2}\right]+W \bar{z}_{+} z_{-}^{2}
$$

with the equation for $z_{-}$obtained by interchanging $z_{+}$and $z_{-}$. The variables $z_{+}, z_{-}$and $\mu, \phi$ represent state and control variables, all of which are zero at the bifurcation point. In this case the null space is parameterized by $\left(z_{+}, z_{-}\right) \in$ $\mathbb{C}^{2}$, and the actions of the group are given by

$$
\begin{gathered}
R\left(z_{+}, z_{-}\right)=\left(-i z_{-}, i z_{+}\right), \quad S\left(z_{+}, z_{-}\right)= \pm\left(z_{-}, z_{+}\right), \\
T_{\theta}\left(z_{+}, z_{-}\right)=e^{i \theta}\left(z_{+}, z_{-}\right) .
\end{gathered}
$$

By defining

$$
\begin{aligned}
& z_{+}=\alpha_{1}+\alpha_{4}+i\left(\alpha_{2}-\alpha_{3}\right), \\
& z_{-}=\alpha_{1}-\alpha_{4}+i\left(\alpha_{2}+\alpha_{3}\right)
\end{aligned}
$$

and changing to these coordinates in $\mathbb{C}^{2}$, we obtain the normal form (50) at cubic order. We remark that although the work of Swift involves analyzing a dynamical normal form (i.e., $\dot{z}_{+}$replaces 0 on the left-hand side of (50)), all results regarding branching of periodic solutions carry over to our steady state case. In order to get dynamical information as well (for example, branching of quasiperiodic solutions), it is necessary to perform a center manifold reduction of the original equations.

For our case, it follows from equations (54) and (55) (in the Supplement section) that the parameters in (50) are given by

$$
\begin{aligned}
\mu & :=\lambda / 2, \\
\phi & :=\tau+d_{0} c / 2 \lambda,
\end{aligned}
$$

implying that $(\mu, \phi)$ is uniquely determined by $(\lambda, \tau)$. The nondegeneracy conditions for determinacy of directions of branching (i.e., not including their stability) are:

$$
\begin{aligned}
R_{u}:= & \operatorname{Re}(U+V / 2+W / 2) \neq 0 \\
R_{v}:= & \operatorname{Re}(U+V / 2-W / 2) \neq 0 \\
R_{w}:= & \operatorname{Re}(U+V) \neq 0 \\
& |V|^{2} \neq|W|^{2} \neq|\operatorname{Re}(V \bar{W})| .
\end{aligned}
$$

The real variables $R_{u}, R_{v}$ and $R_{w}$ determine the branching of the three solutions with the three isotropy subgroups with 2-dimensional fixed point spaces; the Vertex, Edge and Rotating waves referred to by Swift [34]. These are periodic solutions branching from the trivial solution with the maximal symmetry types indicated in Table 1 . The symmetry of vertex (resp. edge) waves is the reflection $R$ (resp. $S$ ). The symmetry of rotating waves relates to its name, 
TABLE 1. The quantities $R_{u}, R_{v}, R_{w}$ determine the branching of solutions with the three maximal isotropy types as the parameter $\mu=\lambda / 2$ varies. The $\tilde{\mathbf{D}}_{2}$ correspond to symmetries generated by reflection in two planes coupled with a temporal phase shift of half a period for one of them.

\begin{tabular}{|l|c|c|c|}
\hline Name & & $\left(z_{+}, z_{-}\right)$ & Symmetry \\
\hline Edge oscillation & $-\mu / R_{u}$ & $z_{+}=z_{-}$ & $\tilde{\mathbf{D}}_{2}(1)$ \\
Vertex oscillation & $-\mu / R_{v}$ & $z_{+}=i z_{-}$ & $\tilde{\mathbf{D}}_{2}(2)$ \\
Rotating wave & $-\mu / R_{w}$ & $z_{-}=0$ & $\tilde{\mathbf{Z}}_{4}$ \\
\hline
\end{tabular}

i.e., the rotation generated by $R \cdot S$ combined with a temporal phase shift of one quarter of a period.

Of special interest in this system is the possible existence of branches of solutions with submaximal isotropy, i.e., branches not predicted by the equivariant Hopf branching lemma of Golubitsky and Stewart [22]. In this system, there can be such solutions with no symmetry bifurcating from the trivial solution if the following condition is fulfilled:

$$
|V|^{2}>|W|^{2}>|\operatorname{Re}(V \bar{W})| .
$$

We concentrate on computing the Hopf bifurcation of the $(1,2):(2,1)$ spatial modes for the Brusselator equations with $A_{0}=1$, several values of $d_{0}$ and using two different (homogeneous) boundary conditions.

3.3.1. Neumann boundary conditions. In order to validate the method, we chose first to calculate the results for Neumann boundary conditions, and verify them against the analytical results obtained in [10]. All the computations are done with double precision in the finite difference methods and using a trapezoidal integration rule with adapted weights for the points on edges and corners. The values of $U, V$, and $W$ in the reduced bifurcation equation (50) were obtained for various values of the discretization parameter at Hopf bifurcation with $A=1, d_{0}=1 / 5$, and as can be seen in Table 2 . Table 3 shows what these values of the coefficients imply for the various quantities determining the branching behavior. In this case, we can verify that the assumptions of 3-determinacy and stability are satisfied for small enough $h$; the nondegeneracy conditions (51) are satisfied. There is excellent $h^{2}$-convergence of the numerical predictions to the exact results. For example, if we denote the error of numerical approximations of $R_{u}$ as a function $\operatorname{Err}\left(h, R_{u}\right)$, the first column of Table 3 shows $\operatorname{Err}\left(1 / 10, R_{u}\right)=0.0202, \operatorname{Err}\left(1 / 20, R_{u}\right)=0.004, \operatorname{Err}\left(1 / 40, R_{u}\right)=0.00102$, which in turn indicates the $h^{2}$-convergence. As can be seen from Table 3, for Neumann boundary condition Hopf bifurcation of the $(1,2):(2,1)$ modes, all branches of solutions exist only for $\mu$ (and therefore $\lambda$ ) positive. We can also see that the conditions for existence of solutions with submaximal symmetry are satisfied. 
TABLE 2. The results with Neumann boundary conditions for the $(1,2):(2,1)$ Hopf bifurcation at $A=1, d_{0}=1 / 5$. The $h$ refers to the step size for discretization, and the reduced equation is determined by the three complex coefficients $U$, $V$ and $W$. The predictions are calculated via fourth-order polynomial extrapolation in $h$. The exact results are taken from Ashwin and Mei [10].

\begin{tabular}{|l|ccc|}
\hline$h$ & $U$ & $V$ & $W$ \\
\hline $1 / 10$ & $-1.40293-0.646856 i$ & $0.811598+0.252318 i$ & $-0.0618543+0.169199 i$ \\
$1 / 20$ & $-1.42061-0.625768 i$ & $0.802736+0.225045 i$ & $-0.0495027+0.187299 i$ \\
$1 / 30$ & $-1.42335-0.621785 i$ & $0.800934+0.220424 i$ & $-0.0470687+0.190293 i$ \\
$1 / 40$ & $-1.42428-0.620388 i$ & $0.800295+0.218836 i$ & $-0.0462092+0.191317 i$ \\
\hline pred. & $-1.42545-0.618593 i$ & $0.799470+0.216815 i$ & $-0.0451025+0.192616 i$ \\
\hline \hline exact & $-1.42545-0.618590 i$ & $0.799467+0.216817 i$ & $-0.0450990+0.192614 i$ \\
\hline
\end{tabular}

TABLE 3. These are the real quantities for Neumann boundary conditions and determine the direction and amplitude of branching. They are shown for various values of the discretization parameter. Note that the nondegeneracy conditions (51) are satisfied for the exact solution and all the listed approximations. Also note that the condition $|V|^{2}>$ $|W|^{2}>|\operatorname{Re}(V \bar{W})|$ for the existence of submaximal solutions is satisfied.

\begin{tabular}{|l|cccccc|}
\hline$h$ & $R_{u}$ & $R_{v}$ & $R_{w}$ & $|V|^{2}$ & $|W|^{2}$ & $\operatorname{Re}(V \bar{W})$ \\
\hline $1 / 10$ & -1.02806 & -0.966210 & -0.591337 & 0.722356 & 0.0324544 & -0.0075087 \\
$1 / 20$ & -1.04399 & -0.994490 & -0.617873 & 0.695031 & 0.0375316 & 0.0024132 \\
$1 / 30$ & -1.04642 & -0.999357 & -0.622424 & 0.690083 & 0.0384272 & 0.0042464 \\
$1 / 40$ & -1.04724 & -1.00103 & -0.623990 & 0.688362 & 0.0387376 & 0.0048861 \\
\hline pred. & -1.04827 & -1.00316 & -0.625983 & 0.686160 & 0.0391346 & 0.0057052 \\
\hline \hline exact & -1.04826 & -1.00361 & -0.625983 & 0.686158 & 0.0391344 & 0.0057069 \\
\hline
\end{tabular}

3.3.2. Dirichlet boundary conditions. The only part of the reduction that needs to be changed for the imposition of Dirichlet boundary conditions $\left(u_{1}=u_{2}=0\right.$ on $\partial \Omega$ after shifting) is in the solution of the linear problems detailed in the Appendix. Summarizing the results in Tables 4 and 5, we can say that the branches of rotating waves bifurcate in the direction $\lambda<0$ while the other two are still bifurcating in the direction $\lambda>0$. Note that in all the computations, the condition for existence of solutions with submaximal symmetry are not satisfied. 
TABLE 4. The results, this time for Dirichlet boundary conditions; $h$ refers to the step size for discretization, and the reduced equations are determined by the three complex coefficients $U, V$, and $W$. The predicted values are calculated via extrapolation of the results for $h=1 / 10, \ldots, 1 / 40$ to $h=0$.

\begin{tabular}{|l|ccc|}
\hline$h$ & $U$ & $V$ & $W$ \\
\hline $1 / 10$ & $-1.09323-0.720547 i$ & $1.29119-0.018280 i$ & $-0.173970-0.068473 i$ \\
$1 / 20$ & $-1.10613-0.716250 i$ & $1.28505-0.056069 i$ & $-0.183272-0.057232 i$ \\
$1 / 30$ & $-1.10866-0.715232 i$ & $1.28371-0.063449 i$ & $-0.184924-0.054831 i$ \\
$1 / 40$ & $-1.10957-0.714853 i$ & $1.28323-0.066101 i$ & $-0.185504-0.053953 i$ \\
\hline pred. & $-1.11078-0.714341 i$ & $1.28257-0.069612 i$ & $-0.186258-0.052777 i$ \\
\hline
\end{tabular}

TABLE 5. The real quantities determining the direction and amplitude of branching for Dirichlet boundary conditions at various values of the discretization parameter.

\begin{tabular}{|l|cccccc|}
\hline$h$ & $R_{u}$ & $R_{v}$ & $R_{w}$ & $|V|^{2}$ & $|W|^{2}$ & $\operatorname{Re}(V \bar{W})$ \\
\hline $1 / 10$ & -0.534623 & -0.360652 & 0.197957 & 1.66750 & 0.034954 & -0.223377 \\
$1 / 20$ & -0.555247 & -0.371974 & 0.178915 & 1.65450 & 0.036864 & -0.232305 \\
$1 / 30$ & -0.559269 & -0.374344 & 0.175052 & 1.65196 & 0.037203 & -0.233912 \\
$1 / 40$ & -0.560712 & -0.375208 & 0.173654 & 1.65104 & 0.037322 & -0.234477 \\
\hline pred. & -0.562622 & -0.376363 & 0.171793 & 1.64984 & 0.037478 & -0.235217 \\
\hline
\end{tabular}

3.3.3. Varying $d_{0}$. Table 6 shows an example of predicted real branching coefficients for $(1,2):(2,1)$ mode Hopf bifurcations with Dirichlet boundary conditions, for a range of values of $d_{0}$, with $A=1$.

TABLE 6. By changing $d_{0}$ with $A=1$, we display the predicted real quantities determining branching at Hopf bifurcation with Dirichlet boundary conditions. These are calculated by extrapolating the results for $h=1 / 10, \ldots, 1 / 40$ to $h=0$. Note that there are higher codimension bifurcations occurring as we change $d_{0}$; for example, $R_{w}$ changes sign near $d_{0}=0.17$ implying that the branch of rotating waves changes criticality nearby. When $R_{w}=0$, the branching is determined by fifthorder terms.

\begin{tabular}{|l|cccccc|}
\hline$d_{0}$ & $R_{u}$ & $R_{v}$ & $R_{w}$ & $|V|^{2}$ & $|W|^{2}$ & $R e(V \bar{W})$ \\
\hline 0.02 & -0.79909 & -0.80675 & -0.27259 & 1.3938 & 0.0014754 & 0.027640 \\
0.04 & -0.77077 & -0.76450 & -0.25075 & 1.2848 & 0.0011589 & 0.0090780 \\
0.06 & -0.75175 & -0.73094 & -0.23063 & 1.2167 & 0.0013566 & -0.0086017 \\
0.08 & -0.73854 & -0.70214 & -0.20973 & 1.1805 & 0.0021347 & -0.026628 \\
0.1 & -0.72865 & -0.67513 & -0.18580 & 1.1724 & 0.0036326 & -0.046176 \\
0.12 & -0.71953 & -0.64686 & -0.15605 & 1.1918 & 0.0060858 & -0.068579 \\
0.14 & -0.70772 & -0.61322 & -0.11631 & 1.2410 & 0.0098723 & -0.095559 \\
0.16 & -0.68738 & -0.56750 & -0.059525 & 1.3258 & 0.015597 & -0.12954 \\
0.18 & -0.64722 & -0.49729 & 0.027399 & 1.4563 & 0.024249 & -0.17417 \\
0.2 & -0.56262 & -0.37636 & 0.17179 & 1.6498 & 0.037478 & -0.23521 \\
\hline
\end{tabular}




\section{ACKNOWLEDGMENTS}

We thank Jacques Furter for helpful conversations. P.A. was supported by the Royal Society European Exchange Program (UK) and M.Z. was supported by the DFG (Germany). We also acknowledge support from a European Community Laboratory Twinning Grant during completion of this work.

\section{BIBLIOGRAPHY}

1. E. L. Allgower, P. Ashwin, K. Böhmer, and Z. Mei, Liapunov-Schmidt reduction for a bifurcation problem with periodic boundary conditions on a square domain, Exploiting Symmetry in Applied and Numerical Analysis (E. L. Allgower, K. Georg, R. Miranda, eds.), Lectures in Appl. Math., vol. 29, Amer. Math. Soc., Providence, RI, 1993, pp. 11-22.

2. E. L. Allgower and K. Böhmer, Resolving singular nonlinear equations, Rocky Mountain J. Math. 18 (1988), 225-268.

3. E. L. Allgower, K. Böhmer, K. Georg, and R. Miranda, Exploiting symmetry in boundary element methods, SIAM J. Numer. Anal. 29 (1992), 534-552.

4. E. L. Allgower, K. Böhmer, and Z. Mei On a problem decomposition for semi linear nearly symmetric elliptic problems, Parallel Algorithms for Partial Differential Equations (W. Hackbusch, ed.), Vieweg Verlag, Braunschweig, 1991, pp. 1-17.

5. —_ A complete bifurcation scenario for the 2d-nonlinear Laplacian with Neumann boundary conditions on the unit square, Bifurcations and Chaos: Analysis, Algorithms, Applications (R. Seydel, F. W. Schneider, T. Küpper, and H. Troger, eds.), Birkhäuser Verlag, Basel, 1991, pp. 1-18.

6. P. Ashwin, High corank mode interactions on a rectangle, Bifurcation and Symmetry (E. L. Allgower, K. Böhmer, and M. Golubitsky, eds), Internat. Ser. Numer. Anal., vol. 104, Birkhäuser, Basel, 1992, pp. 23-33.

7. _ Ph.D. thesis, Math. Institute, University of Warwick, 1991.

8. P. Ashwin and Z. Mei, Normal form for Hopf bifurcation of partial differential equations on the square, submitted to Nonlinearity, 1994.

9. P. Ashwin, K. Böhmer, and Z. Mei, A numerical Liapunov-Schmidt method for finitely determined problems, Exploiting Symmetry in Applied and Numerical Analysis (E. L. Allgower, K. Georg, and R. Miranda, eds.), Lectures in Appl. Math., vol. 29, Amer. Math. Soc., Providence, RI, 1993, pp. 49-69.

10. P. Ashwin and Z. Mei, Liapunov-Schmidt reduction at Hopf bifurcation of the Brusselator equations on a square, University of Warwick, 1992, preprint.

11. K. Böhmer, Developing a numerical Lyapunov-Schmidt method, Bericht zum Fachbereich Mathematik der Philipps-Universität Marburg, 1990.

12. _ On a numerical Lyapunov-Schmidt method for operator equations, Computing 53 (1993), 237-269.

13. K. Böhmer and Z. Mei, On a numerical Lyapunov-Schmidt method, Computational Solutions of Nonlinear Systems of Equations (E. L. Allgower and K. Georg, eds.), Lectures in Appl. Math., vol. 26, Amer. Math. Soc., Providence, RI, 1990, pp. 79-98.

14. F. Brezzi, J. Rappaz, and P. A. Raviart, Finite dimensional approximation of nonlinear problems, Part I: Branches of nonsingular solutions, Numer. Math. 36 (1980), 1-25; Part II: Limit points, Numer. Math. 37 (1981), 1-28; Part III: Simple bifurcation points, Numer. Math. 38 (1981), 1-30.

15. J. D. Crawford, Normal forms for driven surface waves, Physica D 52 (1991), 429-457.

16. M. Dellnitz, Computational bifurcation of periodic solutions in systems with symmetry, IMA J. Numer. Anal. 12 (1992), 429-455.

17. C. C. Douglas and J. Mandel, An abstract theory for the domain reduction method, Computing 43 (1992), 75-96. 
18. H. Esser, Stabilitätsungleichungen für Diskretisierungen von Randwertaufgaben gewöhnlicher Differentialgleichungen, Numer. Math. 28 (1977), 69-100.

19. E. Gekeler, On trigonometric collocation in Hopf bifurcation, Bifurcation and Symmetry (E. L. Allgower, K. Böhmer, and M. Golubitsky, eds.), Internat. Ser. Numer. Anal., vol. 104, Birkhäuser, Basel, 1992, pp. 147-156.

20. K. Georg and R. Miranda, Exploiting symmetry in solving linear equations, Bifurcation and Symmetry (E. L. Allgower, K. Böhmer, and M. Golubitsky, eds.), Internat. Ser. Numer. Anal., vol. 104, Birkhäuser, Basel, 1992, pp. 157-168.

21. M. Golubitsky and D. Schaeffer, Groups and singularities in bifurcation theory, Vol. 1, Appl. Math. Sci., vol. 51, Springer, New York, 1986.

22. M. Golubitsky and I. Stewart, Hopf bifurcation in the presence of symmetry, Arch. Rational Mech. Anal. 87 (1985), 107-165.

23. M. Golubitsky, I.N Stewart, and D. Schaeffer, Groups and singularities in bifurcation theory, Vol. 2, Appl. Math. Sci., vol. 69, Springer, New York, 1988.

24. M.G.M. Gomes, Steady-state mode interactions in rectangular domains, M.Sc. thesis, Math. Institute, University of Warwick, 1989.

25. R.D. Grigorieff, Zur Theorie linearer approximationsregulärer Operatoren, I und II, Math. Nachr. 55 (1973), 233-249 and 251-263.

26. W. Hackbusch, Theorie und Numerik elliptischer Differentialgleichungen, Teubner Verlag, Stuttgart, 1986.

27. J. Martinet, Singularities of smooth functions and maps, LMS lecture notes 58, CUP, 1982.

28. Z. Mei, Path following around Corank-2 bifurcation points of a semi-linear elliptic problem with symmetry, Computing 47 (1991), 69-85.

29. I. Prigogine and P. Glansdorff, Structure, stabilité et fluctuations, Masson, Paris, 1971.

30. H. J. Reinhard, Analysis of approximation methods for differential and integral equations, Springer-Verlag, Berlin, Heidelberg, Tokyo, New York, 1985.

31. E. Stiefel and A. Fässler, Gruppentheoretische Methoden und ihre Anwendung, Teubner Verlag, Stuttgart, 1979.

32. F. Stummel, Diskrete Konvergenz linearer Operatoren, I, Math. Ann. 190 (1970), 45-92; II, Math. Z. 120 (1971), 231-264; III, Proc. Oberwolfach 1971, Internat. Ser. Numer. Anal., vol. 20, Birkhäuser, Basel, 1972, pp. 196-216.

33. Pures Appl. 21 (1976), 63-96.

34. J.W. Swift, Hopf bifurcation with the symmetry of the square, Nonlinearity 1 (1988), 333377.

35. G. Vainikko, Funktionsanalysis der Diskretisierungsmethoden, Teubner Texte zur Mathematik, Teubner, Leipzig, 1976.

36. A. Vanderbauwhede, Local bifurcation and symmetry, Res. Notes in Math., vol. 75, Pitman, Boston, 1982.

Mathematics Institute, University of WaRwick, Coventry CV4 7AL, UNITEd Kingdom

E-mail address: pba@maths .warwick.ac.uk

FB Mathematik, Universität Marburg, Lahnberge, D-35032 Marburg, Germany

E-mail address: boehmer Omathematik.uni-marburg. de

FB Mathematik, Universität Marburg, Lahnberge, D-35032 Marburg, Germany and Department of Mathematics, Xian Jiaotong University, XI'AN 710049, People's Republic OF CHINA

E-mail address: meizhen@mathematik.uni-marburg.de 


\title{
Supplement to
}

\section{A NUMERICAL LIAPUNOV-SCHMIDT METHOD WITH APPLICATIONS TO HOPF BIFURCATION ON A SQUARE}

\author{
PETER ASHWIN, KLAUS BÖHMER, AND MEI ZHEN
}

\section{Computation of the Liapunov-Schmidt Reduction}

We provide here the details of the computations performed to provide the reduced bifurcation equations for Brusselator Hopf bifurcation. We use splittings and null space projections as defined in $\S 3$. For $\lambda, \tau$ in (37)-(39) and for any

$$
\eta:=(z, \lambda, \tau)=\left(\sum_{i=1}^{4} \alpha_{i} \psi_{i}, \lambda, \tau\right) \in \operatorname{Ker}\left(\partial_{u} \Phi_{0}\right) \times \mathbb{R}^{2}=\operatorname{Ker}\left(\Phi_{0}^{\prime}\right)
$$

we find that the truncated bifurcation equations to second order are given by

$$
\begin{aligned}
B_{2}(z, \lambda, \tau)= & (I-\hat{Q}) j_{2} R\left(\sum_{i=1}^{4} \alpha_{i} \psi_{i}, \lambda, \tau\right) \\
= & (I-\hat{Q})\left\{\left(\begin{array}{cc}
1 & 0 \\
-1 & 0
\end{array}\right) \lambda \sum_{i=1}^{4} \alpha_{i} \psi_{i}+\left(\begin{array}{c}
1 \\
-1
\end{array}\right)\left[\frac{B_{0}}{A_{0}}\left(\sum_{i=1}^{4} \alpha_{i} \psi_{i}^{1}\right)^{2}\right.\right. \\
& \left.\left.+2 A_{0}\left(\sum_{i=1}^{4} \alpha_{i} \psi_{i}^{1}\right)\left(\sum_{i=1}^{4} \alpha_{i} \psi_{i}^{2}\right)\right]-\tau \partial_{i} \sum_{i=1}^{4} \alpha_{i} \psi_{i}\right\}
\end{aligned}
$$

where we have used the notation $\psi_{i}=\left(\psi_{i}^{1}, \psi_{i}^{2}\right)^{T}$ and $j_{2}$ truncates to the polynomial of degree 2 in the Taylor expansion of $\Phi$ at $(0,0,0)$ with respect to $\alpha_{i}, \lambda$ and $\tau$. In view of the orthogonality properties of $\left\{\phi_{k l}\right\}$, we can write

$$
\begin{aligned}
& B_{2}(z, \lambda, \tau)=(I-\hat{Q})\left[\left(\begin{array}{cc}
1 & 0 \\
-1 & 0
\end{array}\right) \lambda \sum_{i=1}^{4} \alpha_{i} \psi_{i}-\tau \partial_{t} \sum_{i=1}^{4} \alpha_{i} \psi_{i}\right] \\
= & \sum_{j=1}^{4}\left\langle\psi_{j}^{*},\left(\begin{array}{c}
1 \\
-1
\end{array}\right) \lambda \sum_{i=1}^{4} \alpha_{i} \psi_{i}^{1}-\tau \partial_{t}\left(\sum_{i=1}^{4} \alpha_{i} \psi_{i}\right)\right\rangle \psi_{j} \\
= & \frac{1}{2}\left\{\left[\left(\alpha_{1}-\alpha_{2}\left(P_{11}-P_{12}\right)\right) \lambda-2 \tau \alpha_{2}\right] \psi_{1}+\left[\left(\left(P_{11}-P_{12}\right) \alpha_{1}+\alpha_{2}\right) \lambda+2 \tau \alpha_{1}\right] \psi_{2}\right. \\
& \left.+\left[\left(\alpha_{3}-\alpha_{4}\left(P_{11}-P_{12}\right)\right) \lambda-2 \tau \alpha_{4}\right] \psi_{3}+\left[\left(\left(P_{11}-P_{12}\right) \alpha_{3}+\alpha_{4}\right) \lambda+2 \tau \alpha_{3}\right] \psi_{4}\right\} \\
= & 0,
\end{aligned}
$$



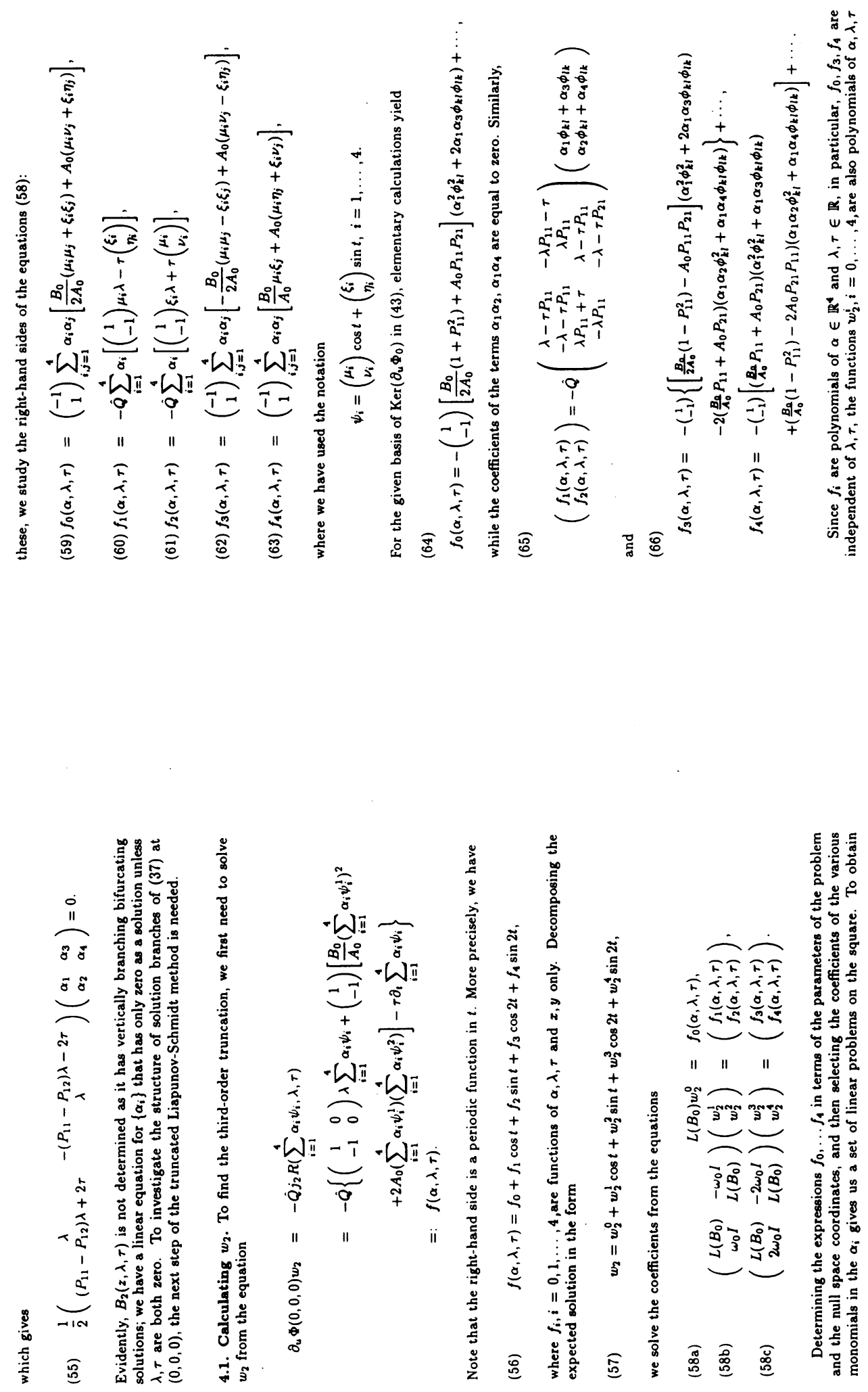

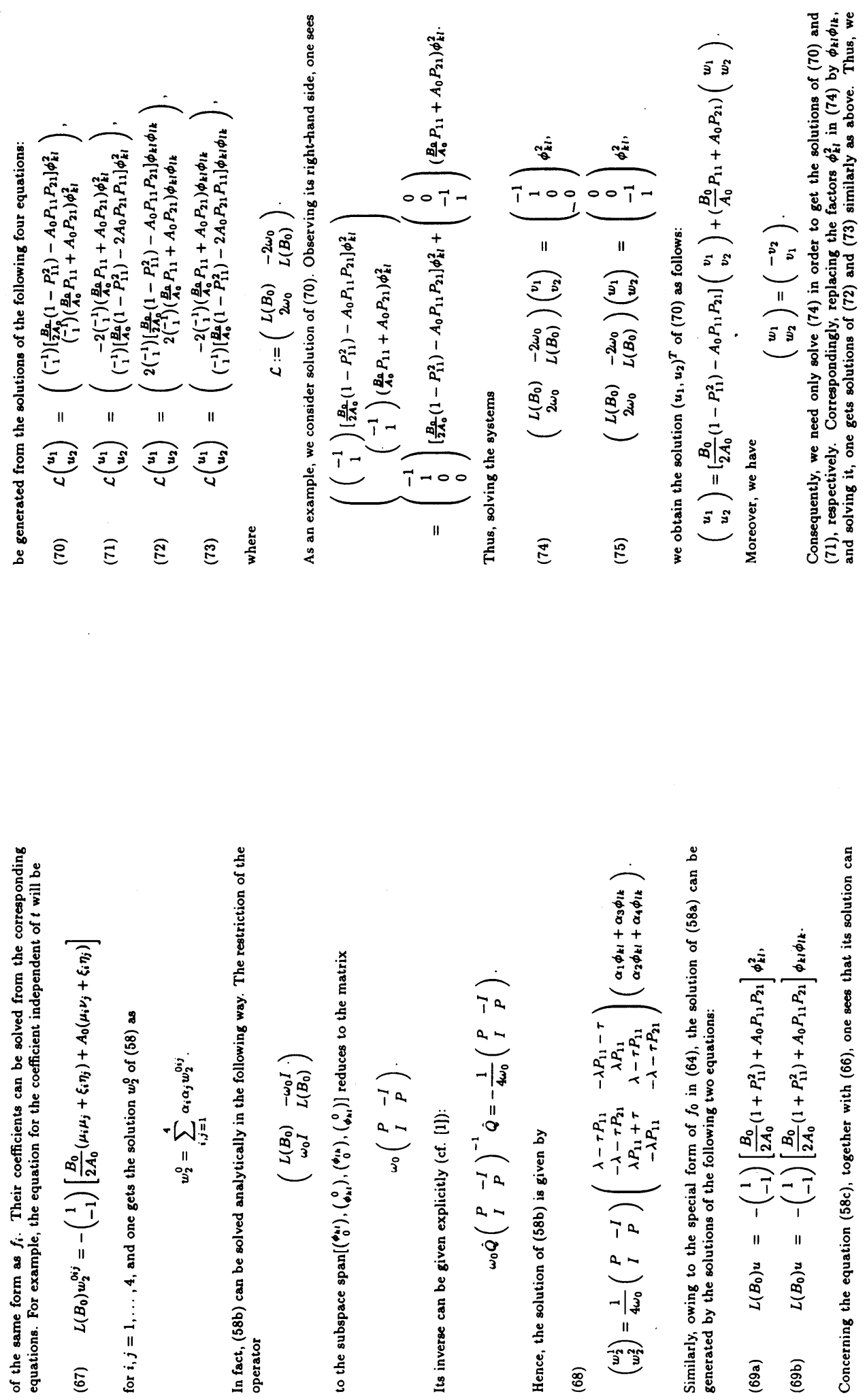

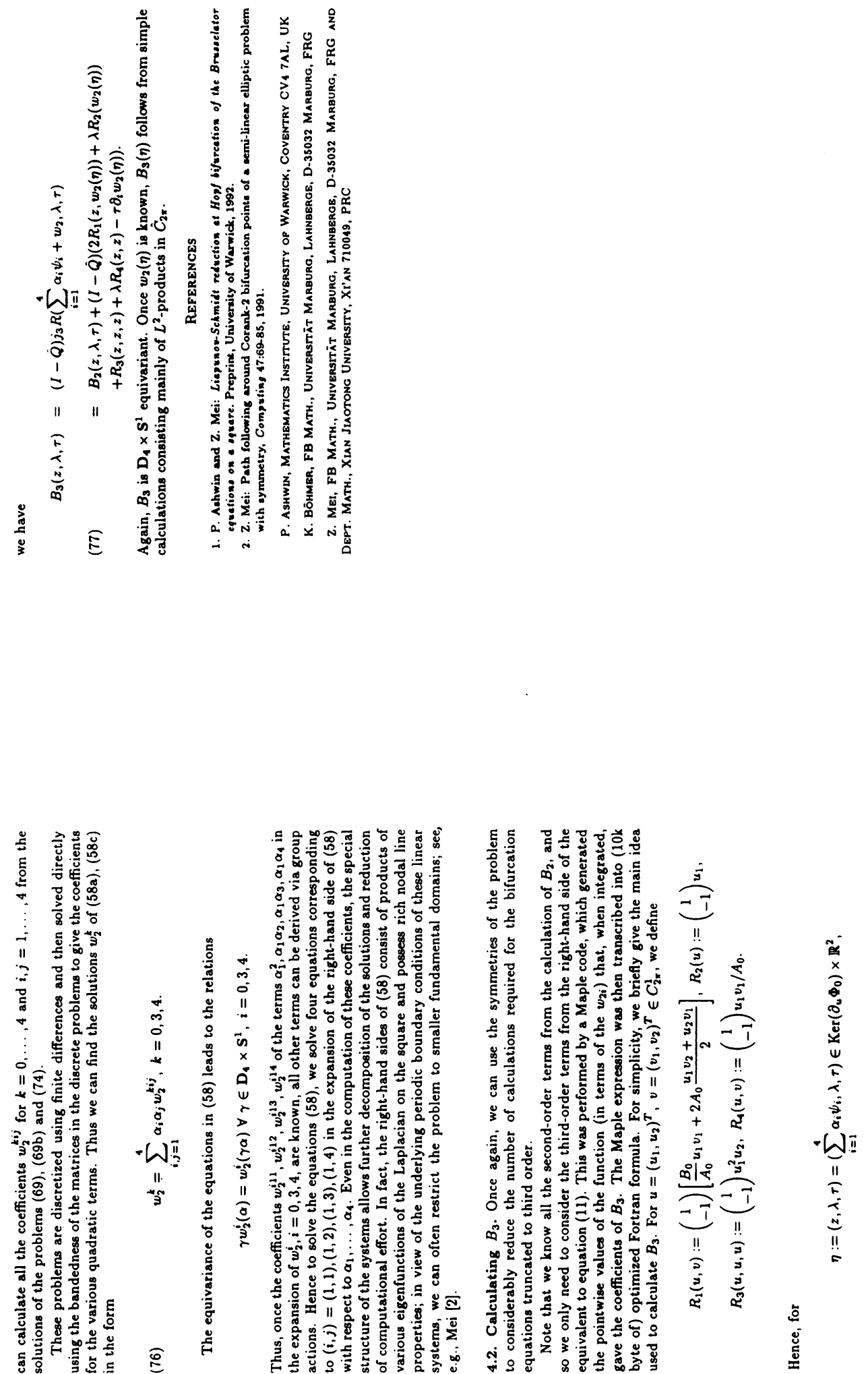